III. Aufgrund der vorstehenden Ausführungen bietet die Berufung offensichtlich keine hinreichende Aussicht auf Erfolg. [...]

DOI: 10.1007/s00350-014-3876-z

\section{Anmerkung zu OLG Koblenz, Beschl. v. 26.2.2014 - 5 U 1441/13 (LG Koblenz)}

\section{Volker Großkopf}

Im pflegerischen Umfeld zählen Schadensersatzansprüche aus Sturzereignissen vor Patienten zu den häufigsten Klagegründen ${ }^{1}$. Dies ist darauf zurückzuführen, dass bei einem Patientensturz auf der Ebene der Sorgfaltspflichtverletzung in der Regel die Beweiserleichterung des voll beherrschbaren Herrschafts- und Organisationsbereiches angewendet wird ${ }^{2}$. Die beweiserleichternde Wirkung des voll beherrschbaren Herrschafts- und Organisationsbereich ist bei einer Transfer- und Transportmaßnahme allerdings nur dann zu bejahen, wenn die Gefahrensituation - in welcher sich der Patient befand - hinreichend konkret war und sich aus der Gefahrenlage gesteigerte Fürsorge- und Obhutspflichten ergaben ${ }^{3}$. Diese können in der Regel nur durch den Einsatz von hinreichend qualifiziertem Personal oder durch den Einsatz entsprechender Hilfsmittel und Gerätschaften erfüllt werden. Denn nur hierdurch kann die identifizierte konkrete Gefahrensituation tatsächlich auch sach- und fachgerecht beherrscht werden ${ }^{4}$. Im vom OLG Koblenz entschiedenen Sachverhalt handelt es sich zwar um eine Transportmaßnahme, aber eine konkrete Gefahrenlage, aus welcher sich gesteigerte Fürsorge- und Obhutspflichten ergeben hätten, lag nach Auffassung des Senats gerade nicht vor, so dass die Beweislast für die Sorgfaltspflichtverletzung bei der Klägerseite verblieb.

Um im Falle eines Schadens nicht dem Vorwurf eines schuldhaften Sorgfaltspflichtverstoßes ausgesetzt zu sein, ist es für die Beklagtenseite von großer praktischer und juristischer Relevanz, dass sich das Handeln des eingesetzten Personals am anerkannten und gesicherten Qualitätsstandard der Wissenschaft und Forschung orientiert. Bei drohender Sturzgefahr liefert ärztlicherseits die Leitlinie der Arbeitsgemeinschaft der Wissenschaftlichen Medizinischen Fachgesellschaften (AWMF-Leitlinien-Register Nr. 53/004) aus dem Jahr 2004 den tätigen Ärzten eine Vielzahl von Handlungsempfehlungen. Im Bereich der Pflege wird die anzuwendende fachgerechte Sorgfalt im Umgang mit sturzgefährdeten Personen seit 2005 durch den „Expertenstandard Sturzprophylaxe in der Pflege" des Deutschen Netzwerks für Qualitätsentwicklung in der Pflege (DNQP) repräsentiert ${ }^{5}$. $1 \mathrm{~m}$ Rahmen von Sturzunfällen und der Frage, ob sorgfaltswidrig gehandelt wurde, wird ggf. auch der neueste Expertenstandard des DNQP zur „Erhaltung und Förderung der Mobilität in der Pflege" eine weitere Rolle spielen ${ }^{6}$. Beide Regelwerke bieten dem Verwender zahlreiche Entscheidungshilfen an, die den anerkannten aktuellen Stand der pflegerischen Wissenschaft und Forschung in diesem Handlungsumfeld umschreiben.

1) Großkopf/Schanz, RDG 2006, 2.

2) BGH, NJW 1991, 1540.

3) OLG München, RDG 2009, 129.

4) Vgl. Großkopf, Kompaktwissen Haftpflichtrecht - Die Vertragshaftung in der Pflege 2010, 47.

5) Großkopf/Frohnhofen, RDG 2012, 64

6) Schanz, RDG 2014, 142.

Rechtsanwalt Prof. Dr. iur. Volker Großkopf,

G\&S Verlag GbR, Salierring 48, 50677 Köln, Deutschland

\section{Substantiierungsanforderungen im selbstständigen Beweisverfahren in Arzthaftungssachen}

ZPO §§485 Abs. 2 S. 1 Nr. 2, 567 Abs. 1 Nr. 1

Im selbstständigen Beweisverfahren in Arzthaftungssachen können an die im Rahmen von $\$ 485$ Abs. 2 ZPO zulässigen Fragen keine weitergehenden Substantiierungsanforderungen gestellt werden als im Haftungsprozess selbst. Fragen nach der Ursache eines Schadens sind deshalb zulässig.

OLG Naumburg, Beschl.v. 14. 10.2013 - 1 W 34/13 (LG Halle)

Problemstellung: Einmal mehr hatte sich ein $\mathrm{Ge}-$ richt mit Fragen rund um das selbstständige Beweisverfahren in Arzthaftungssachen zu befassen. Seit Einführung der Regelung des $\$ 485$ ZPO waren sowohl Zulässigkeit als auch Gegenstand dieses Verfahrens in Arzthaftungssachen umstritten (Walter, MedR 2014, 302), es bedurfte erst zweier Judikate des BGH, um ein Stück weit Rechtssicherheit zu schaffen (s. BGH, MedR 2003, 405; BGH, MedR 2014, 302 m. Anm. Walter; dazu auch Katzenmeier, LMK 2014, 355655). Regelmäßig geht es um die vorprozessuale Begutachtung gemäß $\int 485$ Abs. $2 \mathrm{ZPO}$, die ein rechtliches Interesse an der Feststellung eines der gemäß $\ 485$ Abs. 2 S. 1 Nrn. 1 bis 3 ZPO zulässigen Beweisthemen erfordert. Neben der Frage nach dem körperlichen Zustand des Patienten ( $\$ 485$ Abs. 2 S. 1 Nr. 1 ZPO) ist häufig auch die Frage nach dessen Ursache ( $\$ 485$ Abs. 2 S. 1 Nr. 2 ZPO) Gegenstand des Verfahrens. Ein im selbstständigen Beweisverfahren erlangtes Sachverständigengutachten kann gemäß $₫ 493$ Abs. 1 ZPO im sich etwaig anschließenden Haftungsprozess Verwendung finden, was den in den $\$ \int 485 \mathrm{ff}$. ZPO angelegten $\mathrm{Ge}-$ danken der Prozessökonomie zum Ausdruck bringt.

Im vorliegenden Fall ging es nicht um das in Rechtsprechung und Literatur lange Zeit heftig umstrittene Problem, welche Fragen (auch diejenige nach dem Vorliegen eines Behandlungsfehlers und seiner etwaigen Bewertung als ,grob“?) im selbstständigen Beweisverfahren in Arzthaftungssachen zulässig sind, vielmehr hatte das OLG Naumburg über die Anforderungen zu entscheiden, die an eine Substantiierung der Fragen an sich zu stellen sind. Das erstinstanzlich mit dem Verfahren befasste Landgericht hatte einzelne Fragen, die die Antragstellerin klären lassen wollte, für $\mathrm{zu}$ unbestimmt erachtet und war von einer unzulässigen Erhebung eines Ausforschungsbeweises ausgegangen. Dem ist das OLG in seinem Beschluss entgegengetreten. Sowohl die Frage ,Welche Ursache haben die unter Nr. 1 und Nr. 2 aufgeführten Mängel an der Oberkiefer- oder Unterkieferprothese?"“ als auch die Frage ,Welche Ursache hat der unter Nr. 5 aufgeführte krankhafte Zustand der Antragstellerin?“ hat das OLG unter Abänderung des landgerichtlichen Beschlusses für zulässig i. S. von $₫ 485$ Abs. 2 S. 1 Nr. 2 ZPO erachtet, da diesen kein ausforschender Charakter zukomme. Vielmehr gelte auch im Rahmen des selbstständigen Beweisverfahrens, dass an die überhaupt zulässigen Fragen keine weitergehenden Substantiierungsanforderungen gestellt werden könnten als im Haftungsprozess selbst. Eine unzulässige Ausforschung sei im Arzthaftungsverfahren ohnehin nur mit Zurückhaltung anzunehmen, weil an die Sub-

Eingesandt von RiOLG Dr. Tiemann;

bearbeitet von Wiss. Hilfskraft Dr. iur. Dominique Püster,

Universität zu Köln, Institut für Medizinrecht,

Albertus-Magnus-Platz, 50923 Köln, Deutschland 